\title{
APRENDIENDO A MIRAR PROFESIONALMENTE LAS SITUACIONES DE ENSEÑANZA DE LAS MATEMÁTICAS
}

\author{
LEARNING TO NOTICE MATHEMATICS TEACHING SITUATIONS \\ APRENDENDO A OLHAR PROFISSIONALMENTE PARA AS SITUAÇÕES \\ DE ENSINO DA MATEMÁTICA
}

\author{
Claudia Lisete O. Groenwald ${ }^{1}$ (iD) \\ Salvador Llinares ${ }^{2}$ iD \\ ${ }^{1}$ Universidade Luterana do Brasil, Canoas, Brasil \\ ${ }^{2}$ Universidad de Alicante, Alicante, España
}

\begin{abstract}
Recibido: 30/10/2021 - Aceptado: 22/02/2022 - Publicado: 01/03/2022
Remita cualquier duda sobre esta obra a: Claudia Lisete O. Groenwald. Correoelectrónico: claudiag@ulbra.br
\end{abstract}

\begin{abstract}
RESUMEN
Presentamos una caracterización de la competencia docente "mirar profesionalmente las situaciones de enseñanza de las matemáticas” formada por un conjunto de prácticas profesionales (interpretar, anticipar, decidir) a través de ejemplos de investigación y propuestas formativas en tres programas de formación que pueden apoyar su desarrollo. Los ejemplos descritos desde Brasil, España y Costa Rica se centran en aprender a mirar tareas matemáticas para pensar en la planificación, aprender a mirar la propia enseñanza y aprender a interpretar el pensamiento matemático de los estudiantes para justificar nuevas líneas de acción. La conceptualización y desarrollo de la competencia docente "mirar profesionalmente las situaciones de enseñanza de las matemáticas" señalan que es necesario que los profesores aprendan las prácticas profesionales que se vinculan a esta competencia docente. Algunas de estas prácticas son reconocer e interpretar la demanda cognitiva de las tareas y anticipar las respuestas de los estudiantes para decidir nuevas líneas de actuación, en función de la interpretación de las respuestas de los estudiantes y de los sucesos del aula.
\end{abstract}

Palabras clave: Mirar profesionalmente; Aprendizaje docente; Formación de profesores de matemáticas.

\begin{abstract}
This paper characterizes professional noticing as a mathematics teaching competence formed by a set of core practices (interpreting, anticipating, deciding) through different research examples and training proposals from three teacher education programs that can support their development. The examples from Brasil, Spain
\end{abstract}


and Costa Rica focus on preservice mathematics teachers' learning of noticing mathematical tasks when preservice teachers think about planning, noticing the own teaching, and noticing students' mathematical thinking to make teaching decision. The conceptualization of professional noticing and its development suggest that prospective teachers should learn the core practice articulating this teaching competence. Some of these core practices are attending to and interpreting the cognitive demand of mathematical tasks and anticipating students' answers and making decision about what to do next regarding the interpretation of students' mathematical thinking and teaching actions.

Keywords: Professional Noticing; Teacher's Learning; Mathematics Teacher Education.

\section{RESUMO}

Apresentamos uma caracterização da competência docente "olhar profissionalmente para as situações de ensino da matemática” formada por um conjunto de práticas profissionais (interpretar, antecipar, decidir) por meio de diferentes exemplos de pesquisa e propostas formativas, nos programas de formação de professores, que podem apoiar seu desenvolvimento. Os exemplos descritos do Brasil, da Espanha e da Costa Rica se centram em se centram em aprender a olhar as tarefas matemáticas para pensar no planejamento didático, aprender a olhar o ensino e aprender a interpretar o pensamento matemático dos estudantes para justificar novas linhas de ação. A conceituação e o desenvolvimento da competência docente "olhar profissionalmente para as situações de ensino da matemática" indicam que é necessário que os professores desenvolvam práticas profissionais que se vinculem a essa competência docente. Algumas dessas práticas são reconhecer e interpretar a demanda cognitiva das tarefas, antecipar as respostas dos alunos para decidir novas linhas de ação, com base na interpretação das respostas dos alunos e das situações positivas ocorridas em sala de aula.

Palavras-chave: Olhar profissionalmente; Aprendizagem docente; Formação de professores de matemática.

\section{INTRODUCCIÓN}

Este artículo presenta principios e ideas de una línea de investigación en formación de profesores de matemáticas centrada en caracterizar el desarrollo de competencias profesionales en la formación inicial y continua. Presentamos ejemplos de investigaciones realizadas por los grupos de investigación GECEM (Grupo de Estudos Curriculares de Educação Matemática ${ }^{1}$ ) de la Universidade Luterana do Brasil (ULBRA), Canoas, Rio Grande do Sul, Brasil, y GIDIMAT-UA (Grupo de Investigación de Didáctica de la Matemática²), de la Universidad de Alicante, España.

En las reflexiones sobre el aprendizaje profesional de los docentes se destaca el aprendizaje a lo largo de su carrera como un camino hacia la mejora de la práctica pedagógica. Se entiende que el aprendizaje profesional no se da solo en los cursos de formación institucionalizados, sino que permea cada acción pedagógica del profesor, incluidas la planificación de tareas matemáticas, la predicción de qué y cómo se espera que el alumno resuelva una tarea propuesta, y la reflexión (del profesor) sobre la eficacia de lo planificado. Estos procesos ejemplifican ciclos de mejora continua, con planificación, reflexión (tanto individual como con otros profesores) y re-planificación.

\footnotetext{
${ }^{1}$ http://dgp.cnpq.br/dgp/espelhogrupo/17138.

${ }^{2}$ https://web.ua.es/es/gidimat/grupo-de-investigacion-de-didactica-de-la-matematica.html.
} 
Desde esta perspectiva, la enseñanza de las matemáticas se considera una tarea compleja que implica la toma de decisiones que involucran diferentes conocimientos (Ball et al., 2008; Escudero \& Sánchez, 2007) y que requiere que los profesores tengan una comprensión amplia del conocimiento matemático que esperan enseñar y una visión clara de cómo se desarrolla y progresa el aprendizaje de los estudiantes. Es decir, la enseñanza de las matemáticas (entendida como una práctica que debe ser aprendida) requiere comprender lo que los estudiantes necesitan para desarrollarse matemáticamente; de las metas de la formación matemática; y de considerar estas metas para guiar la toma de decisiones durante el proceso de enseñanza (NCTM, 2015). Por tanto, el docente necesita aprender a generar conocimientos e información sobre las situaciones en las que tiene que actuar, para poder tomar decisiones para enseñar.

Según Le Boterf (1994) la competencia no es un estado, es un proceso, la competencia es un saber hacer reconocido, como modelo dinámico que implica la movilización de conocimientos teóricos, procedimentales y experimentales y del saber hacer social. Así, la competencia "mirar profesionalmente las situaciones de enseñanza de las matemáticas” permite al docente de matemáticas dar sentido a lo que está sucediendo en su aula (Llinares, 2013b; Llinares \& Fernández, 2021; Mason, 2002; Scheiner, 2021). Esta competencia debe entenderse como ser capaz de identificar lo que es relevante para el aprendizaje matemático de los estudiantes, interpretándolo para justificar las decisiones de acción de acuerdo con los objetivos planificados (Mason, 2021).

La competencia docente puede entenderse como el conjunto de destrezas que usan los conocimientos de forma relevante en la realización de las actividades profesionales vinculadas a la enseñanza y aprendizaje de las matemáticas. Por ejemplo, identificando los objetivos esperados del alumno, planificando actividades adecuadas al nivel de los estudiantes, eligiendo las metodologías según los contenidos que se impartirán y planificando una evaluación adecuada (Eraut, 1994). En este sentido, la noción de competencia involucra dos procesos: la transferencia de conocimiento y la movilización de recursos disponibles y relacionados con la acción (Silva, 2008).

Para Perrenoud (2000), los recursos son conocimientos procedimentales; conocimientos derivados de la experiencia o recuerdos que se refieren a lo que se está haciendo o lo que se ha visto hacer en situaciones comparables; conocimientos teóricos para movilizar un problema, los procesos involucrados y las estrategias disponibles en este proceso; conocimientos metodológicos que aporten hipótesis para ordenar preguntas, memorizar hechos, comparar hipótesis, complementar y verificar datos, etc.; y conocimientos para buscar información (asesoramiento, ayuda, búsquedas en bases de datos); y conocimientos tácticos y organizativos. Según Perrenoud (2000), el ejercicio de la competencia pasa por operaciones mentales complejas, esquemas de pensamiento que permiten determinar y ejecutar, de forma más o menos eficaz, una acción adaptada a la situación. Desde esta perspectiva, si el docente necesita dar sentido a las situaciones de enseñanza de las matemáticas y comparar lo que ha sucedido con lo que se pretendía (metas) para determinar nuevas líneas de acción, se infiere que una competencia 
fundamental es “mirar profesionalmente las situaciones de enseñanza de las matemáticas”, asumiéndose que esta competencia puede desarrollarse desde la formación inicial y a lo largo de la práctica profesional.

Estos planteamientos justifican el desarrollo de una agenda de investigación con el siguiente objetivo: Investigar características del desarrollo de la competencia docente "mirar profesionalmente las situaciones de enseñanza de las matemáticas” e identificar principios de diseño de los ambientes de aprendizaje en los programas de formación que lo apoyan. Para describir los principios que organizan esta línea de investigación en educación matemática, presentamos algunas concepciones de la competencia docente "mirar profesionalmente las situaciones de enseñanza de las matemáticas". Particularizamos algunas de estas concepciones con ejemplos de investigaciones que permiten mostrar la diversidad de aproximaciones realizadas en Brasil, España y Costa Rica.

En los últimos años han sido realizadas diferentes síntesis de las investigaciones a nivel internacional en esta línea de investigación que han mostrado su relevancia para la educación matemática (Dindyal et al., 2021; Fernández et al., 2018; Schack et al., 2017). Finalmente, en este trabajo y a partir del conjunto de ideas y ejemplos presentados, generamos algunas ideas para desarrollos futuros en esta agenda de investigación.

\section{COMPETENCIA DOCENTE "MIRAR PROFESIONALMENTE LAS SITUACIONES DE ENSEÑANZA DE LAS MATEMÁTICAS"}

Las situaciones de enseñanza de las matemáticas son complejas, estableciéndose relaciones entre los estudiantes, el contenido matemático a ser aprendido y los docentes en contextos sociales determinados. Para mejorar el aprendizaje matemático de los estudiantes en la escuela es necesario tener en cuenta estas relaciones y considerar la práctica docente del profesor. Desde esta perspectiva, la práctica de enseñar matemáticas se puede considerar como un sistema de actividades formado por “prácticas nucleares”, tales como describir e interpretar una situación de enseñanza de las matemáticas, anticipar respuestas de los estudiantes al planificar una lección, interpretar las respuestas de los estudiantes cuando resuelven un problema y gestionar las discusiones matemáticas en el aula, tanto en pequeño grupo como en gran grupo.

La articulación de estas diferentes prácticas ayuda a configurar la manera en la que los docentes de matemáticas razonan en y sobre las situaciones de enseñanza (Groenwald \& Llinares, 2019; Llinares, 2013b; Seibert et al., 2013). Esta manera de entender la enseñanza de las matemáticas como un conjunto de prácticas nucleares se pone de manifiesto en los procesos de razonamiento de los profesores sobre el pensamiento matemático de los estudiantes, sobre las características de las demandas cognitivas de las actividades/tareas en el plan de una lección y en la manera en la que entienden las discusiones matemáticas en la clase. 


\section{CONCEPTUALIZACIÓN}

Una forma de referirse a la articulación de algunas de estas prácticas nucleares es lo que conocemos como la competencia docente "mirar profesionalmente las situaciones de enseñanza de las matemáticas” (Dindyal et al., 2021; Fernández et al., 2018; Llinares et al., 2020; Llinares \& Fernández, 2021; Stahnke et al., 2016). Esta competencia docente puede ser entendida como una colección de prácticas dirigidas a estar en disposición de reconocer diferentes posibilidades de acción, y se apoya en los procesos de razonamiento del profesor usando conocimiento de los dominios científicos que dan soporte a la práctica de enseñar matemáticas (en particular, la Didáctica de las Matemáticas y las Matemáticas). Interpretar las situaciones de la práctica, en nuestro caso la enseñanza de las matemáticas, como un paso necesario para decidir nuevas líneas de actuación docente, implica identificar los elementos relevantes de la situación para el objetivo pretendido (el aprendizaje de las matemáticas por parte de los estudiantes).

En este proceso Mason (2002) distinguió entre describir e interpretar. Describir determinados aspectos de la práctica (incidentes que pueden ser relevantes para el aprendizaje de los estudiantes y la acción del docente), como un proceso diferente de interpretar dichos sucesos usando referencias de los dominios científicos. Distinguir entre describir e interpretar es una manera en la que el docente puede llegar a ser "consciente" de las diferentes posibilidades de acción como una evidencia de la relación entre conocer y hacer.

Sin embargo, aunque existe un cierto consenso en la importancia de la competencia docente "mirar profesionalmente las situaciones de enseñanza de las matemáticas" para desarrollar buenas prácticas docentes, se han generado diferencias en la manera en la que los investigadores la conceptualizan (Jacobs \& Spangler, 2017). Algunos investigadores se han centrado en cómo los docentes y estudiantes para profesor identifican elementos característicos de una situación de enseñanza (conceptualización 1). En una segunda conceptualización los investigadores han considerado los procesos de identificar e interpretar, formando parte de esta competencia docente. Por ejemplo, ante las respuestas de estudiantes a problemas de matemáticas, identificar los elementos matemáticos que aparecen en las estrategias usadas e interpretarlos desde algún modelo teórico de progresión del aprendizaje matemático (Buforn et al., 2020; Fernández et al., 2011).

Finalmente, existe una tercera aproximación a la conceptualización de esta competencia docente que considera los procesos de identificar, interpretar y decidir futuras líneas de acción (conceptualización 3). Por ejemplo, cuando los estudiantes para maestro usan un modelo de progresión de la comprensión del concepto matemático (trayectoria de aprendizaje) para interpretar la comprensión de los estudiantes y poder justificar su propuesta de nuevas tareas para apoyar el aprendizaje de los estudiantes. Por ejemplo, Moreno y colaboradores (Moreno et al., 2021; Sánchez-Matamoros et al., 2018, 2021) describen el uso por parte de los estudiantes para maestro de educación infantil de una trayectoria de aprendizaje de la longitud y su medida como instrumento conceptual para interpretar las estrategias usadas por los niños y justificar las nuevas tareas a proponerles. Por su parte, Ivars et al. (2016, 
2020a, 2020b) describen cómo estudiantes para maestro de educación primaria interpretan la comprensión de los niños del concepto de fracción según un modelo de progresión de la comprensión para justificar las nuevas tareas a ser propuestas.

Una de las características de las investigaciones de la competencia docente "mirar profesionalmente las situaciones de enseñanza de las matemáticas” está relacionada con lo que los estudiantes para profesor miran. Cuando el foco de atención es un tópico matemático particular se generan particularidades que han puesto de manifiesto necesidades que deben ser tenidas en cuenta en esta línea de investigación (Dindyal et al., 2021). Así, algunas de las investigaciones se centran en conceptos o tópicos matemáticos concretos, por ejemplo, la magnitud y medida, las fracciones como en los casos indicados anteriormente (Ivars et al., 2016, 2020; Moreno et al., 2021; Sánchez-Matamoros et al., 2018) o el razonamiento proporcional (Fernández et al., 2011). Estas investigaciones se han centrado en cómo y en qué medida los estudiantes para profesor identifican e interpretan el pensamiento matemático según las evidencias en las resoluciones de los problemas. Existen otras iniciativas en las que los estudiantes para profesor deben "mirar profesionalmente” lecciones (propias o de otros) para identificar e interpretar aspectos relevantes de la enseñanza de las matemáticas y aprender a pensar en estrategias docentes alternativas (Fernández et al., 2020, 2021).

\section{APRENDER A "MIRAR PROFESIONALMENTE"}

El reconocimiento de la importancia de la competencia docente "mirar profesionalmente las situaciones de enseñanza de las matemáticas" ha puesto la atención sobre su desarrollo, es decir, cómo se aprende. Mason (2002) caracterizaba este aprendizaje como un proceso de desarrollo de la sensibilidad de ver "determinados aspectos" como relevantes, lo que permite tener la posibilidad de etiquetarlos y denominarlos de alguna manera, para pensar en formas de actuar. Desde este punto de vista, Mason señala que ser más consciente en las situaciones de enseñanza-aprendizaje puede ser aprendido, considerando cinco maneras: (i) mirar la totalidad, (ii) discerniendo los detalles, (iii) reconocer elaciones, (iv) percibir propiedades, $y$ (v) razonar sobre la base de las propiedades que se han acordado.

En esta misma dirección, van Es y Sherin $(2002,2021)$ subrayan la necesidad de apoyar el desarrollo de una posición de indagación por parte del profesor que subyace al proceso de mirar profesionalmente. Así, enfatizan que identificar los aspectos relevantes en una situación de enseñanza conlleva también a ser conscientes de qué aspectos no son relevantes para el objetivo pretendido. Estos autores inciden en la necesidad de adoptar una perspectiva que problematice la situación para estar en condiciones de realizar inferencias, y tener en cuenta la posibilidad de crear otras situaciones de enseñanza para comprobar las inferencias sobre lo observado, lo que vincula el conocer con el hacer.

La manera en la que los investigadores tienen en cuentas estas características del proceso de aprender la competencia docente "mirar profesionalmente las situaciones de enseñanza de las matemáticas” varía (Fernández \& Choy, 2020). Sin embargo, algunas aproximaciones mantienen 
aspectos comunes. Por ejemplo, considerando los cambios en el discurso de los estudiantes para profesor cuando hablan sobre las situaciones de enseñanza, y entendiendo estos cambios como un proceso de apropiación de conocimiento procedente de las disciplinas científicas que dan apoyo a la práctica de enseñar matemáticas (Ivars et al., 2018, 2020b; Moreno et al., 2021).

Por ejemplo, Ivars et al. (2018) caracterizan el desarrollo de la competencia docente "mirar profesionalmente el pensamiento matemático de los estudiantes de educación primaria”, teniendo en cuenta cómo los estudiantes para maestro usan la información de una trayectoria de aprendizaje del concepto de fracción para interpretar las respuestas de los estudiantes. En esta situación, el uso de la información sobre la comprensión de las fracciones para interpretar las respuestas de los estudiantes parece depender del conocimiento matemático de los estudiantes para maestro. La información sobre la trayectoria de aprendizaje ayudó a los estudiantes para maestro a generar un discurso más detallado en relación con las estrategias usadas por los estudiantes de educación primaria, incluyendo de manera adecuada evidencias para apoyar sus inferencias, al mismo tiempo que evitaban incluir información no relevante. Esta manera de entender el proceso de aprender a "mirar profesionalmente las situaciones de enseñanza de las matemáticas" es explicada por el papel de "andamio" que desempeña la información sobre la trayectoria de aprendizaje del concepto matemático proporcionada a los estudiantes para maestro.

Por otra parte, Moreno y sus colegas (Moreno et al., 2021; Sánchez-Matamoros et al., 2018, 2021) usan las nociones de génesis instrumental y de trayectoria de aprendizaje para explicar cómo los estudiantes para maestro de educación infantil aprenden a mirar profesionalmente el aprendizaje y la enseñanza de las nociones de magnitud y medida. Las diferentes maneras en la que los estudiantes para maestro usaban la información sobre la trayectoria de aprendizaje de la magnitud y la medida en educación infantil son explicadas teniendo en cuenta la generación de dos esquemas de acción instrumentada (uno para interpretar el aprendizaje de los estudiantes y otro para decidir cómo continuar la enseñanza). Desde esta perspectiva, aprender a "mirar profesionalmente" se comprende como el resultado de las interacciones de los estudiantes para maestro con la información proporcionada por la trayectoria de aprendizaje (entendida como un artefacto cultural) al resolver tareas específicas en el programa de formación docente (problemas profesionales).

La trayectoria de aprendizaje se convierte en un instrumento cuando los estudiantes para maestro la usan para razonar sobre las situaciones de enseñanza. En este caso, los niveles de desarrollo de la competencia docente "mirar profesionalmente las situaciones de enseñanza de las matemáticas" vienen dados por el uso paulatinamente más integrado de los elementos de la trayectoria de aprendizaje en el discurso generado por los estudiantes para maestro. Los resultados obtenidos indican que los estudiantes para maestro tienen dificultades en apropiarse de la estructura conceptual de la trayectoria de aprendizaje de la magnitud y medida (por ejemplo, el ver la necesaria dependencia entre el aprendizaje del concepto de magnitud y el aprendizaje de la idea de medida de la magnitud). 
Globalmente, estas investigaciones muestran tres características de aprender la competencia “mirar profesionalmente las situaciones de enseñanza de las matemáticas”. En primer lugar, es un proceso que conlleva ser capaz de identificar los elementos relevantes en una situación de enseñanza (por ejemplo, en las estrategias empleadas por los estudiantes al resolver los problemas de matemáticas), pero también en no tener en cuenta los elementos no relevantes. Esta característica del proceso de aprender la competencia docente indica una mayor sensibilidad hacia lo que puede ser relevante para el aprendizaje de las matemáticas. Es decir, progresando en la manera en la que se perciben aspectos comunes en respuestas diferentes de los estudiantes, evidenciando el reconocimiento de relaciones y propiedades (viendo lo particular como un ejemplo de lo general).

En segundo lugar, se pone de manifiesto la necesidad de que los estudiantes para maestro posean un vocabulario específico que les permita hablar con sentido de lo que es identificado como relevante. En este caso, la información de la trayectoria de aprendizaje y su estructura conceptual (por ejemplo, cómo se describe la progresión de la comprensión de los estudiantes) desempeñan un papel de andamiaje para focalizar la atención y proporcionar los términos necesarios para desarrollar un discurso profesional.

Finalmente, en tercer lugar, las decisiones de cómo continuar y la justificación dada apoyada en las inferencias realizadas sobre la comprensión de los estudiantes permiten determinar el rango de posibles acciones que los estudiantes para maestro pueden generar (razonando sobre la base de las propiedades que se asumen explican las estrategias de los estudiantes). Estas características del proceso de aprender la competencia docente "mirar profesionalmente las situaciones de enseñanza de las matemáticas” se apoyan en llegar a problematizar las situaciones de enseñanza.

\section{EJEMPLOS}

Las investigaciones sobre la competencia docente "mirar profesionalmente las situaciones de enseñanza de las matemáticas” y su desarrollo varían en lo que es observado (el pensamiento matemático de los estudiantes, las interacciones entre los profesores y los estudiantes durante las lecciones, o las interacciones de los profesores con los materiales curriculares). En esta sección describimos ejemplos de investigaciones en esta agenda que permiten ilustrar lo que los investigadores se preguntan y las características del conocimiento generado para la formación de maestros y profesores de matemáticas. Los ejemplos se centran en aprender a mirar las tareas matemáticas para pensar en la planificación, aprender a mirar la propia enseñanza y aprender a interpretar el pensamiento matemático de los estudiantes para justificar nuevas líneas de acción.

\section{APRENDERA MIRAR LAS TAREAS MATEMÁTICAS PARA PENSAR SOBRE LA PLANIFICACIÓN}

Aprender a planificar la enseñanza de las matemáticas implica analizar el potencial de las tareas matemáticas para el objetivo de aprendizaje pretendido. Los estudiantes para profesor deben aprender a 
identificar los elementos relevantes en diferentes tareas matemáticas para determinar su adecuación en el plan de la lección, y deben interpretarlos desde los dominios científicos que dan soporte a la práctica de planificar la lección de matemáticas (Bernabeu et al., 2021; Choy et al., 2017; De Sousa et al., 2020). Aquí lo que se quiere indicar es que, al diseñar los módulos formativos, el formador de profesores transforma el conocimiento desde el dominio científico que da soporte a la práctica de enseñar matemáticas, y lo transforma en contenido del programa de formación. Este contenido del programa de formación es el que el estudiante para profesor debe aprender a usarlo para convertirlo en parte de su conocimiento profesional. Por eso hablamos de entornos de aprendizaje donde se desarrollan diferentes destrezas vinculadas a la competencia docente "mirar profesionalmente". Por ejemplo, los estudiantes para profesor deben usar el conocimiento de matemáticas y el conocimiento sobre la demanda cognitiva de las tareas (Stein et al., 1996) cuando analizan las tareas matemáticas o diseñan y adaptan nuevas tareas usando e integrando tecnología. Al respecto, Llinares (2013a, p. 121) indicaba que:

La elección y adaptación de las tareas matemáticas es una actividad profesional importante del profesor, asi como la gestión que hace durante la enseñanza (Penalva; Llinares, 2011). El análisis del profesor de las tareas matemáticas para planificar una lección para sus alumnos moviliza su conocimiento de matemáticas en relación con el aprendizaje de los alumnos. La manera en la que el estudiante para profesor identifica lo relevante de la tarea matemática y cómo lo vincula al aprendizaje pretendido con sus alumnos a los objetivos curriculares es una manifestación de la competencia docente (Morris et al., 2009). Es decir, la competencia docente para analizar tareas matemáticas viene determinada por la manera en la que el estudiante para profesor identifica la actividad matemática que la tarea puede potenciar en sus alumnos (la idea de demanda cognitiva de la tarea matemática).

En este contexto, describimos dos ejemplos. En primer lugar, una propuesta realizada en el programa de formación de profesores de matemáticas de educación secundaria (Universidad de Alicante, España) dirigida a aprender a analizar las tareas matemáticas al planificar una lección (Figura 1). El proceso de interpretar la "demanda cognitiva" de las tareas (lo que exige la tarea al resolutor) (Stein et al., 1996) que aparecen en este conjunto de páginas web ayuda a los estudiantes para profesor a generar procesos de razonamiento integrando conocimiento de geometría, sobre las características de las tareas (la demanda cognitiva) y sobre el desarrollo del pensamiento geométrico (tareas de memorización, procedimientos sin conexión, procedimientos con conexión y tareas que exigen conjeturar, organizar información y establecer relaciones entre representaciones). En este caso, los niveles de demanda cognitiva considerados fueron cuatro: tareas de memorización, procedimientos sin conexión, procedimientos con conexión y tareas que exigen conjeturar, organizar información y establecer relaciones entre representaciones (Stein et al., 1996). 


\section{Figura 1}

Tarea profesional en el programa de formación de profesores centrada en el desarrollo de la competencia docente vinculada al análisis de tareas matemáticas (Llinares, 2013a, p. 122)

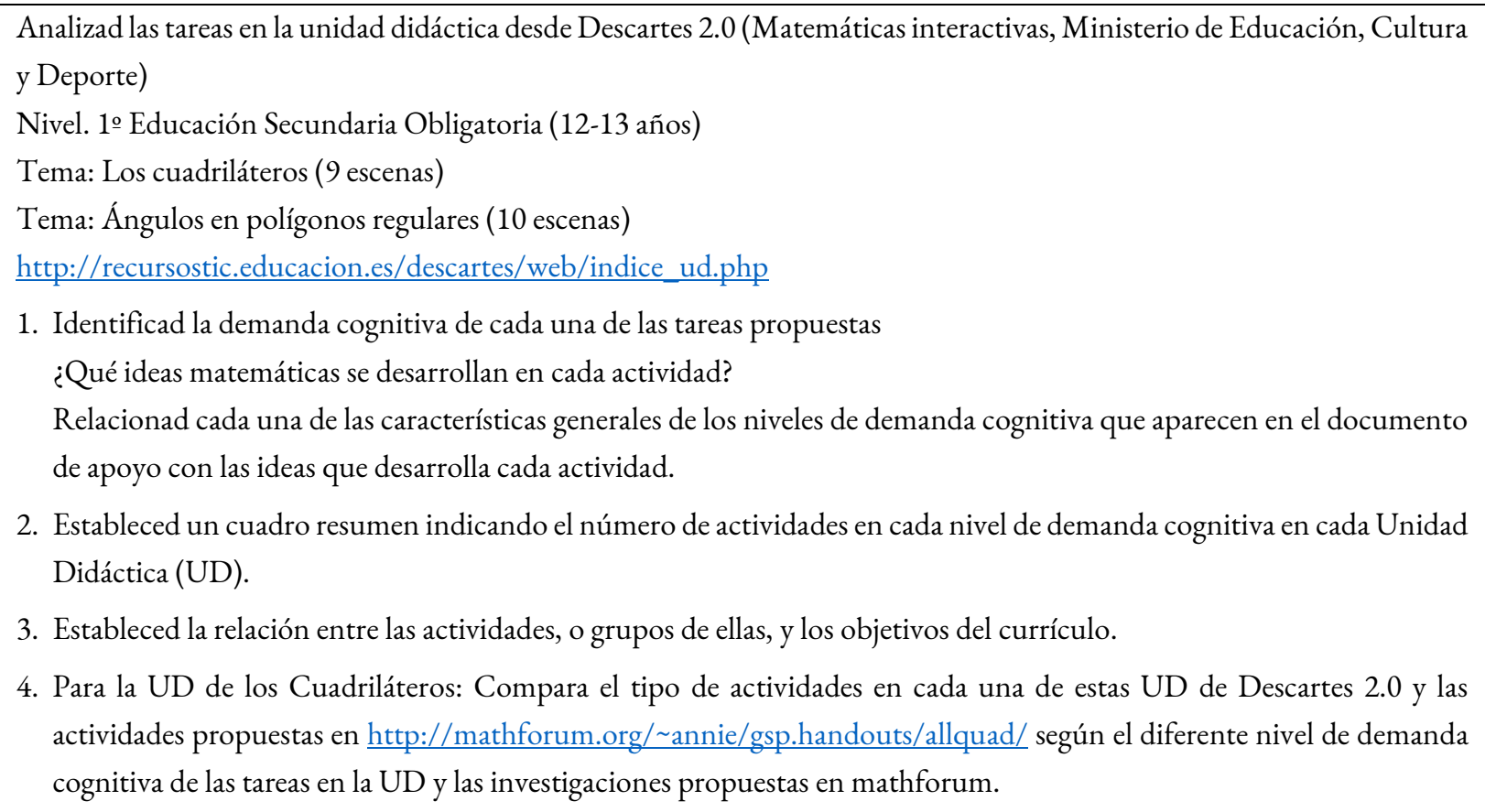

Nota. Los enlaces usados en esta tarea eran de acceso libre cuando se diseñaron los entornos de aprendizaje, lo que puede hacer que en algunos momentos no funcionen en los años posteriores.

Estos entornos de aprendizaje en los programas de formación reflejan tres características. Primero, integran recursos didácticos a ser usados en las lecciones de matemáticas como registros de la práctica. Segundo, proporcionan el referencial teórico para generar los procesos de interpretación (información sobre la noción de demanda cognitiva y sobre el desarrollo del pensamiento geométrico) (Jaime \& Gutiérrez, 1990; Stein et al., 1996). Finalmente, proporcionan “preguntas guía” para focalizar la atención. Estas tres características (registros de la práctica, información teórica y preguntas guía) permiten crear los ambientes que favorecen que los estudiantes para profesor generen aproximaciones investigativas, problematizando la situación de planificar la enseñanza de las matemáticas para realizar inferencias. Además, la información proporcionada en el programa de formación les dota de un vocabulario específico para hablar sobre los aspectos de la práctica, lo que les ayuda a interpretar y justificar posibles líneas de acción futura.

Un segundo ejemplo se centra en oportunidades para que los estudiantes para profesor discutan y reflexionen en grupo al planificar una lección usando recursos tecnológicos. El contexto es un grupo de estudiantes de Matemáticas (Universidade Luterana do Brasil - ULBRA), analizando el objeto de aprendizaje construido con el software GeoGebra para el desarrollo de algunos aspectos del pensamiento geométrico, tales como visualizar, representar, conjeturar, argumentar y probar en relación con el 
teorema de Pitágoras. La Figura 2 presenta el objeto construido y su potencialidad para apoyar el discurso matemático en el aula en la resolución de tareas investigativas.

\section{Figura 2}

\section{Objeto de aprendizaje para el teorema de Pitágoras (http://ppgecim.ulbra.br/laboratorio)}

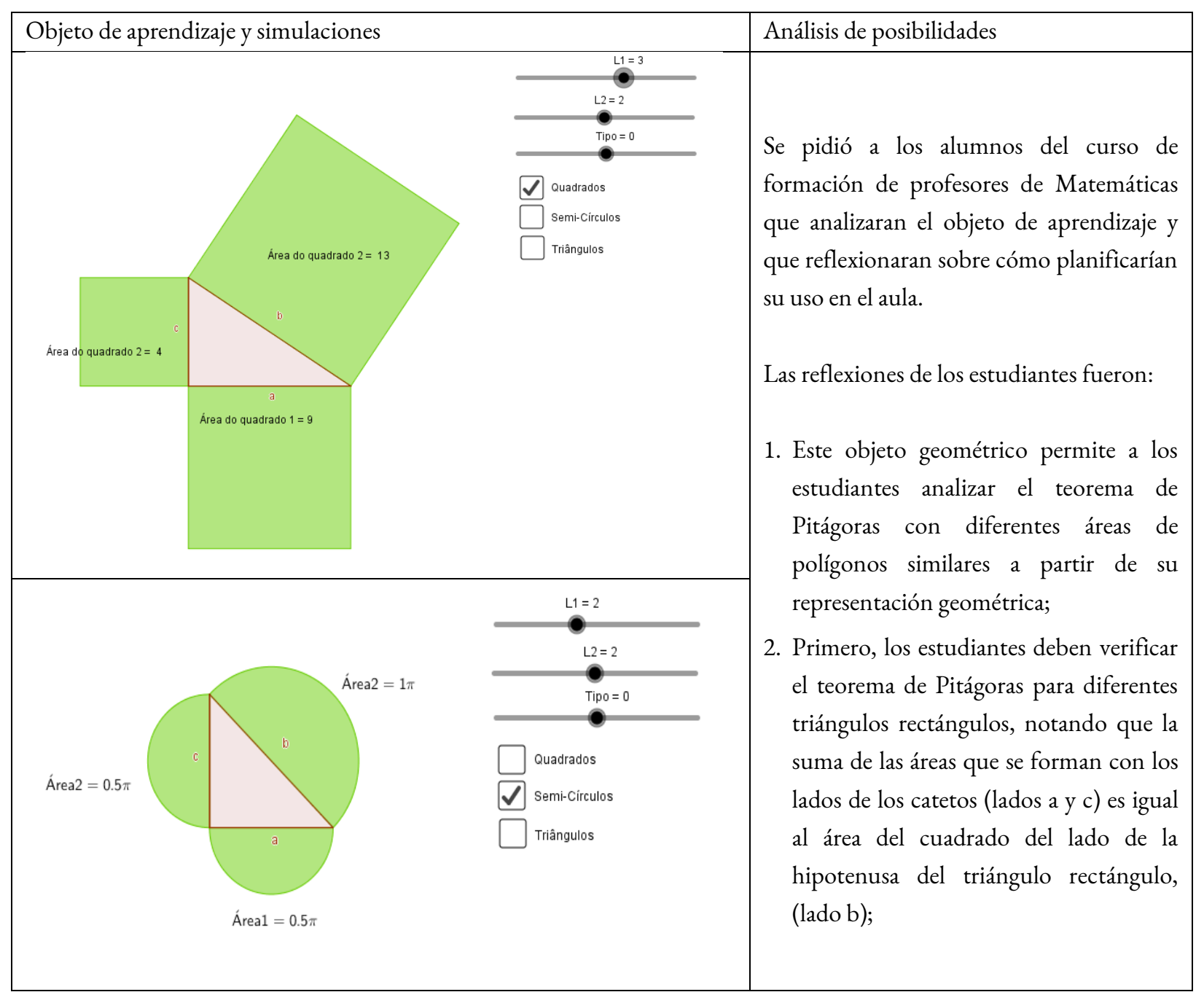




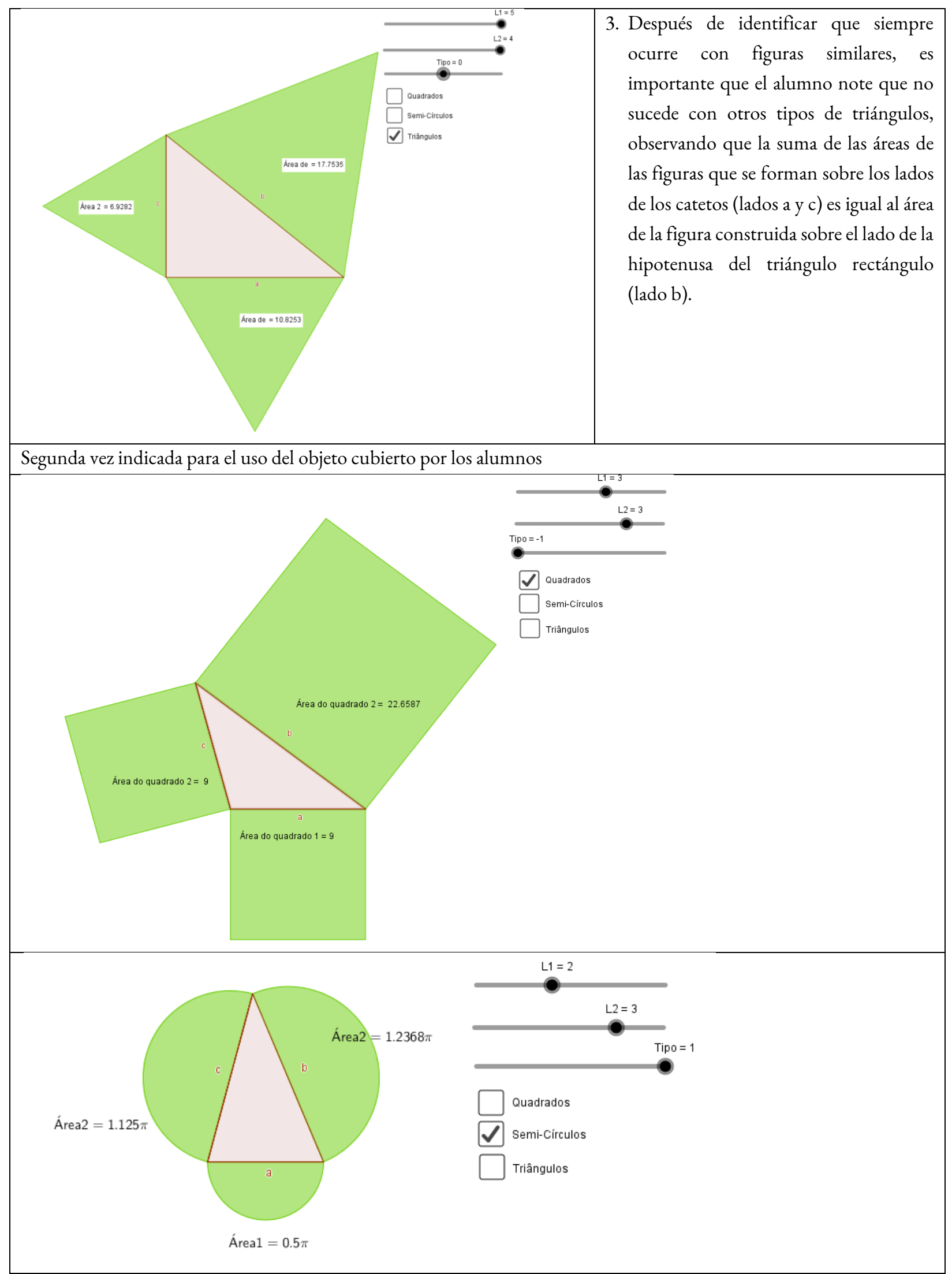




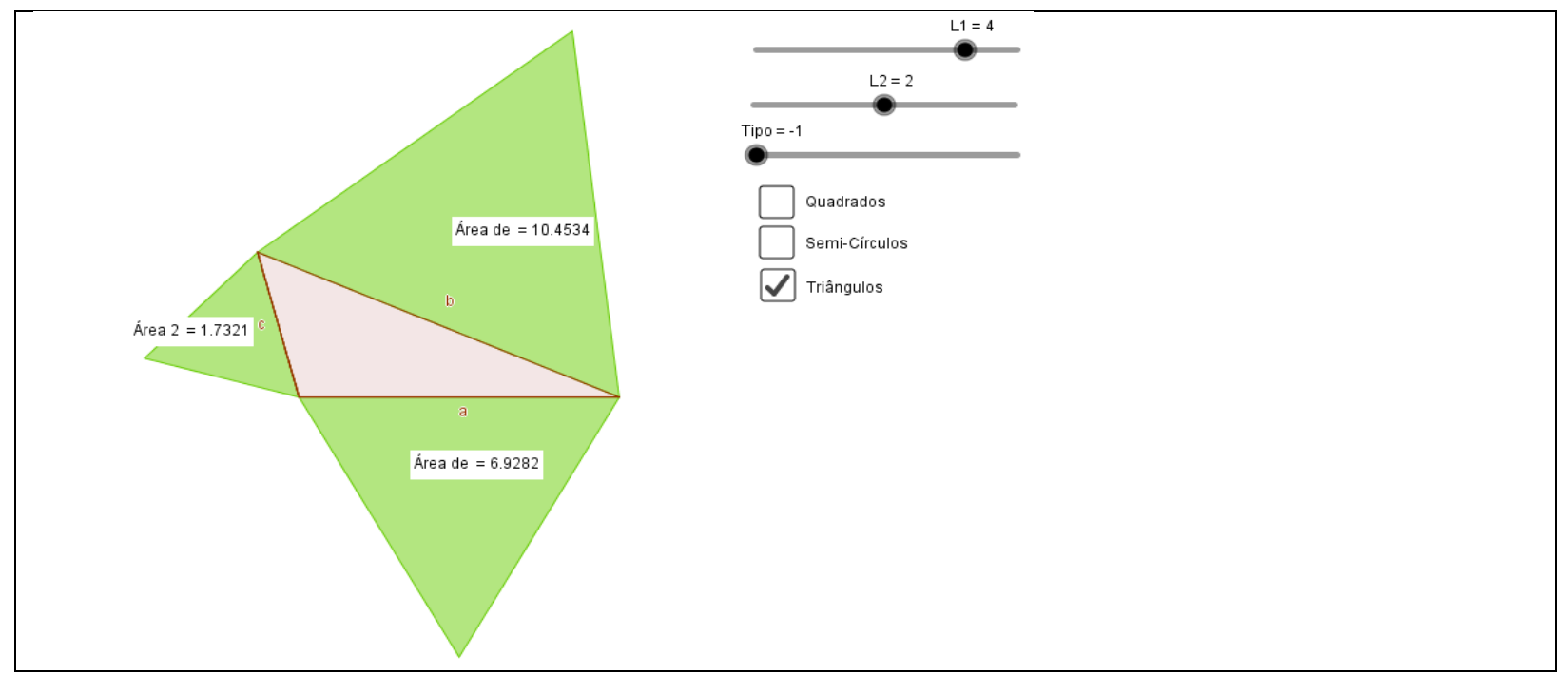

Los resultados del análisis de cómo los estudiantes para profesor interpretaron la demanda cognitiva de las tareas generadas con este objeto de aprendizaje construido integrando tecnología (en este caso usando GeoGebra) mostraron las dificultades de considerar el planteamiento abierto de la propuesta. Es decir, sobre la naturaleza investigativa de las tareas. De esta manera, el uso que anticipan los estudiantes para profesor de este tipo de tareas no tuvo en cuenta las posibilidades de explorar o conjeturar propiedades. Es decir, no consideraron que el objeto geométrico podía permitir a los estudiantes explorar para formular hipótesis y conjeturas e inferir conclusiones. Así, el uso de este objeto de aprendizaje se redujo a que los estudiantes de educación secundaria solo comprobaran el teorema de Pitágoras. Es decir, los estudiantes para profesor reducían el potencial de la tarea investigativa. Estos resultados muestran la necesidad de generar oportunidades durante la formación inicial de profesores de aprender a reconocer el potencial de las tareas investigativas que se pueden integrar en la planificación de las lecciones. Para este fin, la discusión en grupos para analizar las tareas puede ayudar a los estudiantes para profesor a identificar las variables relevantes en el objeto de aprendizaje, e interpretarlo desde su potencial para favorecer en el aula la generación de ambientes investigativos que ayuden a los estudiantes a desarrollar un discurso matemático adecuado en contextos para conjeturar y validar las conjeturas.

Los resultados de las investigaciones del desarrollo de la mirada profesional sobre las tareas matemáticas generadas a partir de estas propuestas formativas mostraron las dificultades de los estudiantes para profesor para analizar las actividades geométricas. En particular, los estudiantes para profesor tuvieron dificultades en reconocer relaciones y en percibir propiedades comunes entre las actividades cuando había que generar clases de cuadriláteros o para generalizar el teorema de Pitágoras. Además, resultó difícil para los estudiantes para profesor razonar sobre la base de las propiedades que se habían identificado para decidir la secuencia de las actividades en la planificación. 
La importancia de estos resultados radica en evidenciar que la manera en la que los estudiantes para profesor "ven” las tareas matemáticas determina en qué medida consideran que estas tareas proporcionan oportunidades efectivas para aprender un contenido matemático relevante para sus alumnos, o qué variables de la actividad pueden ser consideradas como potencialmente modificables para adaptar la actividad a algún grupo de alumnos particulares.

\section{APRENDER A MIRAR LA ENSEÑANZA DE LAS MATEMÁTICAS}

Aprender a mirar la enseñanza de las matemáticas en el aula es desafiante para los estudiantes para profesor. La enseñanza de las matemáticas es una práctica compleja con relaciones entre los estudiantes, el contenido matemático y los profesores en un contexto determinado. Aprender a discernir los detalles relevantes en la enseñanza para el aprendizaje de las matemáticas, establecer relaciones (por ejemplo, entre la demanda cognitiva de la tarea y las estrategias empleadas por los alumnos para resolverla) y llegar percibir algunas características de la enseñanza (por ejemplo, considerar la manera en la que el profesor pregunta a sus alumnos y gestiona el discurso matemático en el aula) es un proceso desafiante para los estudiantes para profesor (Humes et al., 2019). En la investigación reportada por Fernández et al. $(2020,2021)$ sobre cómo estudiantes para profesor de educación secundaria aprendían a mirar (pensar de manera reflexiva) su propia enseñanza, en un programa de formación en Costa Rica, los estudiantes para profesor compartían narrativas de su propia enseñanza con sus compañeros durante el periodo de "prácticas de enseñanza” a través de una plataforma en línea y recibían retroalimentación por parte de su tutor y otros compañeros.

Los resultados indican que aprender a mirar la enseñanza se evidenció mediante cambios en cómo los estudiantes para profesor identificaban oportunidades potencialmente relevantes para el aprendizaje de sus alumnos y las aprovechaban. Los estudiantes para profesor describían con más detalle las situaciones, y aprovechaban las oportunidades identificadas indicando acciones específicas que permitían a los estudiantes continuar con su aprendizaje. Estas acciones específicas eran conectadas a la interpretación de la situación (considerando el potencial de las actividades matemáticas propuestas y las estrategias usadas por los estudiantes para resolverla). La manera en la que los estudiantes para profesor proponían y describían acciones específicas, tales como ignorar la respuesta de los estudiantes o reconocerlas, pero continuar con la planificación prevista, mostraba características del proceso de razonamiento de los estudiantes para profesor y los procesos a través de los cuales daban forma a la instrucción a partir de las inferencias realizadas desde lo observado.

De esta manera, la forma que adoptaba la enseñanza a partir de la interpretación dada a lo observado permitía determinar características de cómo los estudiantes para profesor estaban aprendiendo a mirar profesionalmente su enseñanza. En este contexto, escribir narrativas de la propia enseñanza fue un proceso que medió en el aprendizaje de la competencia docente. 


\section{APRENDER A MIRAR EL PENSAMIENTO MATEMÁTICO DE LOS ESTUDIANTES}

Una tarea profesional del profesor es analizar las tareas matemáticas considerando su demanda cognitiva y anticipando posibles respuestas de los estudiantes (Fernández et al., 2014). Por ejemplo, en un proyecto de Formación Continuada de profesores de Matemáticas (Enseñanza Fundamental, Canoas, Brasil), los profesores tenían que seleccionar tareas para la introducción del tema de ecuaciones lineales y su resolución, anticipar posibles respuestas de los estudiantes y luego analizar las respuestas dadas por sus estudiantes para decidir nuevas líneas de acción (replanteando en su caso la secuencia de actividades inicialmente prevista). Los profesores podían usar el significado de "demanda cognitiva de las tareas" (Penalva \& Llinares, 2011) para hacer visibles los elementos relevantes de las tareas e interpretar el nivel de demanda cognitiva, lo que podía justificar su introducción en la planificación.

De la misma forma que en el ejemplo descrito anteriormente, los niveles de demanda cognitiva considerados fueron cuatro: tareas de memorización, procedimientos sin conexión, procedimientos con conexión y tareas que exigen conjeturar, organizar información y establecer relaciones entre representaciones (Stein et al., 1996). La Figura 3 muestra ejemplos de tareas elegidas por los profesores para la introducción del principio aditivo y multiplicativo para la enseñanza de las ecuaciones.

\section{Figura 3}

Tareas matemáticas para La introducción de las ecuaciones en 6o de Enseñanza Fundamental (Actividades adaptadas de libros didácticos analizadas por los profesores de matemática 3 , Damasco et al., 2020).

\begin{tabular}{|c|c|}
\hline 6o año de la escuela primaria & señanza Fundamental en Brasil) \\
\hline Objetivo: Que el alumno desar & el principio aditivo y multiplicativo con Números Naturales positivos y Números Racionales. \\
\hline Demanda Cognitiva & Tarea de matemáticas \\
\hline Nivel 1 & Desarrolle las multiplicaciones de cada miembro de la igualdad: \\
\hline & $36 \times 14=63 \times 8$ \\
\hline Tarea de memorización & a) ¿Qué resultado tiene cada miembro? \\
\hline (Concepto de igualdad e & b) Sumando 4 a cada miembro de la igualdad, ¿qué notas? \\
\hline introducción al principio & c) Restando 2 de cada miembro de la igualdad, ¿qué se percibe? \\
\hline aditivo y multiplicativo con & d) Multiplicando por 11, cada miembro de esta igualdad, ¿qué percibes? \\
\hline expresiones numéricas) & $\begin{array}{l}\text { Se justifica como nivel } 1 \text { porque involucra definiciones previamente aprendidas. } \\
\text { También involucra reproducir exactamente algo visto previamente y lo que se tiene que } \\
\text { reproducir se establece clara y directamente. }\end{array}$ \\
\hline Anticipando las respuestas $\mathrm{d}$ & estudiantes \\
\hline Cada miembro da como resu & lo el número 504. \\
\hline $36 \times 14+4=63 \times 8+4$ & \\
\hline Los resultados siguen siendo & mismos, manteniendo la igualdad \\
\hline $36 \times 14-2=63 \times 8-2$ & \\
\hline Los resultados siguen siend & mismos, manteniendo la igualdad \\
\hline
\end{tabular}

${ }^{3}$ Docentes de Matemáticas que participan en Educación Continuada en la investigación realizada en ULBRA con el tema Ecuaciones en los últimos años de la escuela primaria (Enseñanza Fundamental en Brasil). 


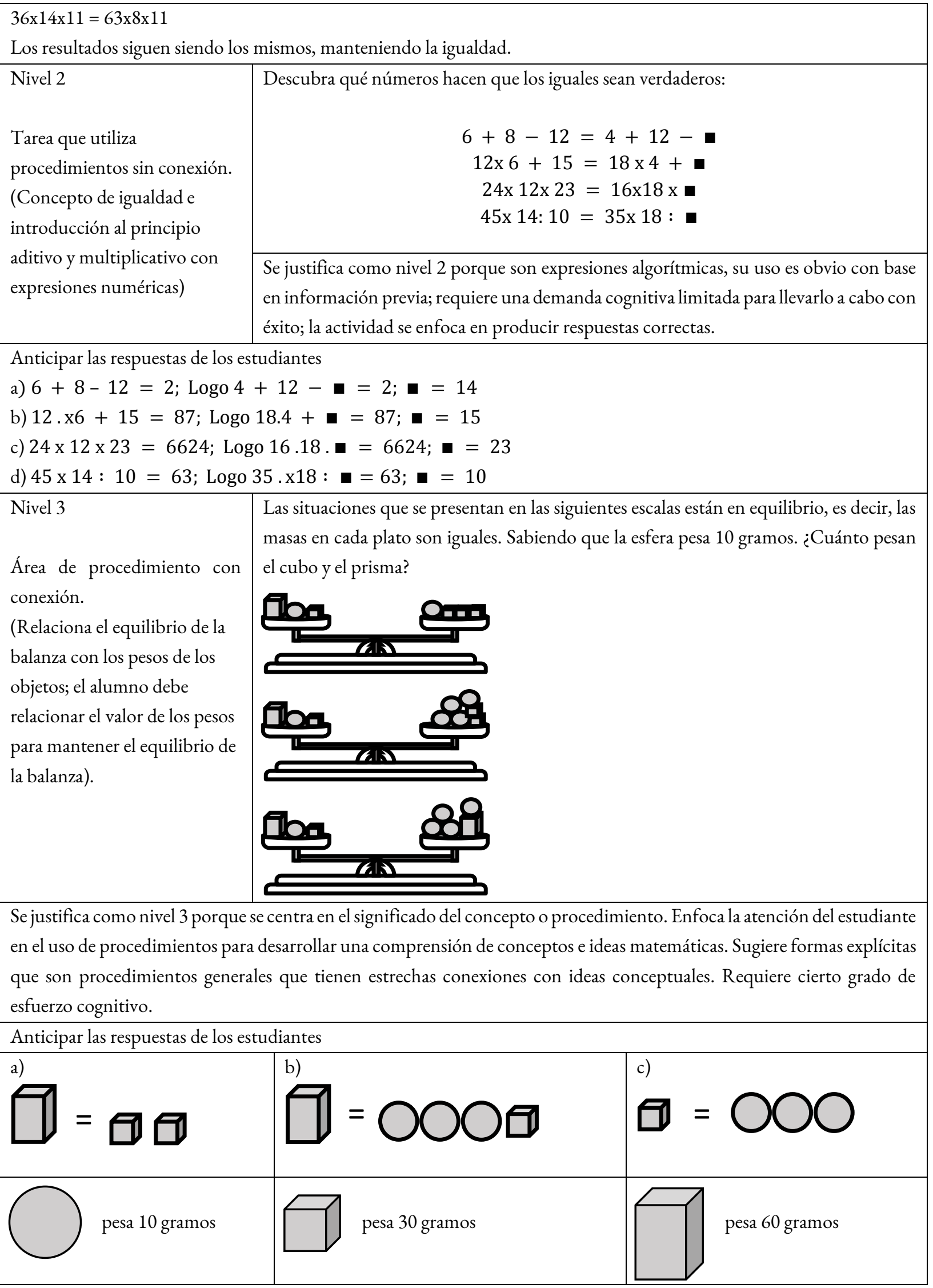




\begin{tabular}{|l|l|}
\hline Nivel 4 & $\begin{array}{l}\text { La siguiente escala está en equilibrio. Los dos cubos tienen la misma medida de masa, } \\
\text { cada prisma tiene una medida de masa igual a } 35 \text { gramos y la esfera tiene una medida de } \\
\text { masa igual a } 72 \text { gramos. ¿Cuál es la medida de masa de cada cubo? }\end{array}$ \\
Tarea que requiere organizar \\
la información para generar \\
un abordaje con éxito. \\
(Principio aditivo y \\
multiplicativo; requiere \\
resolver una ecuación que \\
debe organizarse con los datos \\
del problema.)
\end{tabular}

Los profesores elegían y clasificaban las tareas según su demanda cognitiva y justifican su introducción en la planificación, apoyándose en los conocimientos que los estudiantes debían movilizar para su resolución. Además, los profesores anticipaban posibles respuestas de sus estudiantes a estas tareas para apoyar sus interpretaciones. Después, los profesores usaban estas tareas en clase y analizaban las respuestas de sus estudiantes, lo que les permitía realizar modificaciones en la secuencia inicialmente planteada.

En esta experiencia, los estudiantes respondían correctamente a las tareas de memorización y reproducción de procedimientos sin conexión, aunque tenían dificultades en justificar que la igualdad entre los miembros de la ecuación se mantenía cuando se realizaban operaciones. Esto llevó a los profesores a explicitar, como objetivo de aprendizaje, la necesidad de reforzar el significado de igualdad en una ecuación, ya que los estudiantes operaban de manera separada cada parte de la igualdad (ver Figura 4 para una respuesta de los alumnos). 


\section{Figura 4}

Respuesta a una tarea de memorización (N1)

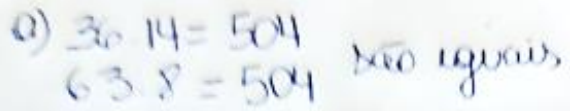

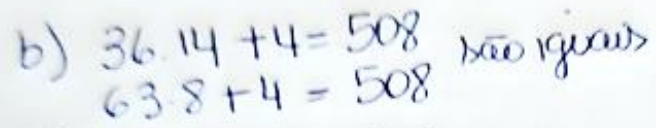

$$
\begin{aligned}
& \text { c) } \begin{array}{l}
36 \cdot 14-2=502 \\
63 \cdot 8-2=502
\end{array} \text { sāo iguais } \\
& \text { d) } \begin{array}{l}
36.14 .11=5544 \\
63.8 \cdot 11=5544
\end{array} \text { sā igiais }
\end{aligned}
$$

En las tareas de procedimientos con conexión (nivel 3), aunque los estudiantes discutían en grupo las resoluciones, tenían dificultades en comunicar la igualdad. Sin embargo, con la mediación del profesor y el uso de la analogía de la balanza de dos brazos era posible desarrollar el significado de la equivalencia entre los dos miembros de la igualdad, facilitando la manera de registrar dicha equivalencia. La Figura 5 muestra la manera en la que los estudiantes registraron la equivalencia entre las dos partes de la ecuación.

\section{Figura 5}

Respuesta de un grupo de estudiantes a una tarea de procedimientos con conexión (nivel 3)

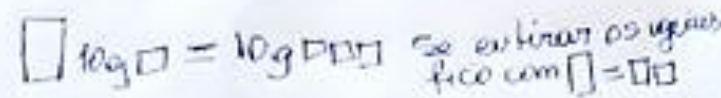

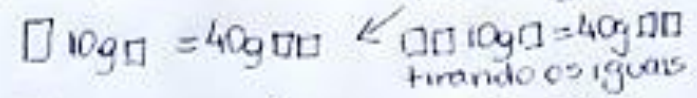

$$
\begin{aligned}
& \square \log n=40 \mathrm{~g} i \longleftarrow \quad \square=309 \\
& \checkmark \log \square=\log \square \square \\
& \text { tirando os igares } \\
& \square=309 \\
& \square=309 \\
& \Pi=\square 0=30 g+30 g=60 g \\
& \text { R: } 0 \text { wbo pesa } 30 \mathrm{~g} \text { e oprisma } 60 \mathrm{~g}
\end{aligned}
$$

En las tareas que los profesores consideraban que reflejaban en mayor medida la actividad matemática (nivel 4), los estudiantes tuvieron mayores dificultades para registrar la igualdad entre las partes de la ecuación. En la respuesta de la Figura 6 (se identifica una respuesta incompleta y con errores), 
podemos observar que los estudiantes consiguen interpretar la igualdad, pero no representan las operaciones correctamente por lo que no aplican el principio aditivo para resolver la igualdad. Los profesores interpretaron esta situación indicando la necesidad de introducir un mayor número de tareas de modelización en las que las ecuaciones muestran las relaciones entre las cantidades, y en las que se tenga que aplicar el principio aditivo para resolver la igualdad.

\section{Figura 6}

Respuesta de un grupo de estudiantes a tareas reflejando actividad matemática (nivel 4)

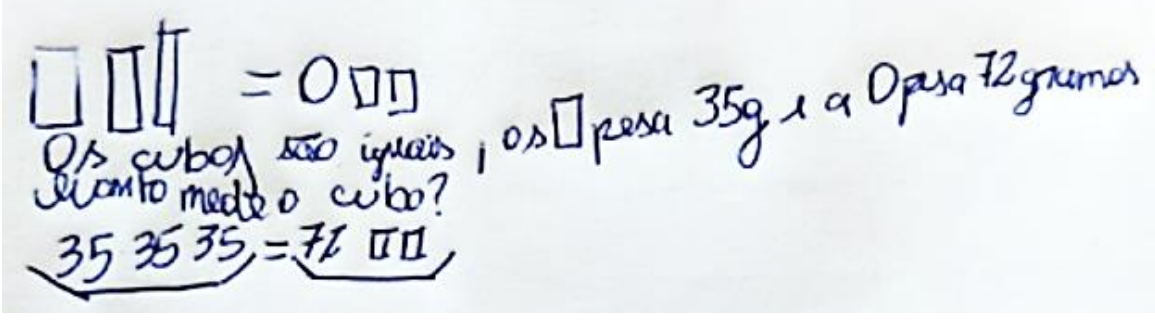

Los resultados de esta investigación muestran que el ciclo formado por la elección de tareas matemáticas y la justificación de la secuencia de las tareas en la planificación de una lección generada por cómo los profesores anticipan posibles respuestas de sus alumnos, junto con el análisis posterior de las respuestas reales que permitía pensar sobre la planificación de la lección, se ha mostrado como un medio potente para el desarrollo de la competencia docente "mirar profesionalmente las situaciones de enseñanza de las matemáticas".

\section{PROSPECTIVA}

Enseñar matemáticas puede ser visto como un sistema de diferentes prácticas profesionales tales como interpretar el pensamiento matemático de los estudiantes, interpretar el potencial matemático de las tareas, planificar secuencias de tareas al pensar en el plan de la lección y/o anticipar posibles respuestas de los estudiantes a las tareas previstas. Estas diferentes prácticas se apoyan en procesos de razonamiento basado en conocimiento de matemáticas y de didáctica de las matemáticas, así como del contexto en el que se desarrolla la enseñanza (Buforn et al., 2020). Recientemente, estos conjuntos de prácticas se consideran que forman parte de la competencia docente del profesor que se denomina "mirar profesionalmente las situaciones de enseñanza de las matemáticas" en la que se evidencia la relación entre conocer y hacer.

La conceptualización de esta competencia docente ha hecho que se realicen aproximaciones a la formación de los profesores centradas en apoyar el desarrollo de estas prácticas (Ivars et al., 2017; Moreno \& Llinares, 2018). Por ejemplo, entendemos que las tareas matemáticas por sí mismas no son suficientes para generar una actividad matemática más significativa en los estudiantes, es decir, no basta con proponer buenas tareas matemáticas para transformar la enseñanza, sino que es necesario que los 
profesores reconozcan y reflexionen sobre el potencial de dichas tareas matemáticas para que puedan ser usadas de una determinada manera.

Desde esta perspectiva, los resultados de las investigaciones sobre el desarrollo de la competencia docente “mirar profesionalmente las situaciones de enseñanza de las matemáticas” señalan que es necesario que los profesores tengan un conocimiento adecuado de los contenidos, pero que también aprendan las prácticas que se vinculan a la competencia docente, tales como reconocer e interpretar la demanda cognitiva de las tareas, anticipar e interpretar las respuestas de los estudiantes y decidir nuevas líneas de actuación.

\section{ACLARATORIAS}

Los autores no tienen conflicto de interés que declarar. La participación de Salvador Llinares en este trabajo ha sido realizada con el apoyo del proyecto Referencia: PID2020-116514GB-I00, Agencia Estatal de Investigación, Ministerio de Ciencia e Innovación, España, y PROMETEO 2017-135, Generalitat Valencia, España. La participación de Claudia Lisete O. Groenwald en este trabajo ha sido realizada con el apoyo del CNPq - Conselho Nacional de Desenvolvimento Científico e Tecnológico con la beca de Productividad Científica PQ-2, 2019-2021.

\section{REFERENCIAS}

Ball, D. L., Thames, M. H., \& Phelps, G. (2008). Content knowledge for teaching: what makes it special? Journal of Teacher Education, 59, 389-407. https://doi.org/10.1177/0022487108324554

Bernabeu, M., Moreno, M., \& Llinares, S. (2021). Anticipating primary school students' answers of hierarchical classifications tasks: features of preservice primary teachers' curricular reasoning. Acta Scientiae. Revista de Ensino de Ciencias e Matemática, 23(6), 121-146. https://doi.org/10.17648/acta.scientiae.6874

Buforn, A., Llinares, S., Fernández, C., Coles, A., \& Brown, L. (2020). Pre-service teachers' knowledge of the unitizing process in recognizing students' reasoning to propose teaching decisions. International Journal of Mathematics Education in Science and Technology, 1-19. https://doi.org/10.1080/0020739X.2020.1777333

Choy, B. H., Thomas, M. O. J., \& Yoon, C. (2017). The FOCUS framework: characterising productive noticing during lesson planning, delivery, and review. En E. O. Schack, M. H. Fisher, \& J. A. Wilhelm (Eds.), Teacher noticing: bridging and broadening perspectives, contexts, and frameworks (pp. 445-466). Springer International Publishing.

https://doi.org/10.1007/978-3-319-46753-5_26 
Damasco, F. C., Groenwald, C. L. O., \& Llinares, S. (2020). A competência docente de observar com sentido situações de ensino e aprendizagem na matemática. En C. T. Kaiber, \& C. L. O. Groenwald (Eds.), Ensino e aprendizagem em ciências e matemática: referenciais, práticas e perspectivas (pp. 7-25). Editora da ULBRA. http://ppgecim.ulbra.br/laboratorio/wp-content/uploads/2020/11/perspectivapratica.pdf

Dante, L. R. (2018). Teláris Matemática. Ática.

De Sousa, J. R., Silva Gusmão, T. C. R., Font, V., \& Lando, J. C. (2020). Task(Re)design to enhance the didactic-mathematical knowledge of teachers. Acta Scientiae. Revista de Ensino de Cienciase Matemática, 22(4), 98-120. https://doi.org/10.17648/acta.scientiae.5711

Dindyal, J., Schack, E., Choy, B. H., \& Sherin, M. G. (2021). Exploring the terrains of mathematics teacher noticing. ZDM Mathematics Education, 53, 1-16.

https://doi.org/10.1007/s11858-021-01249-y

Eraut, M. (1994). Developing professional knowledge and competence. Routledge.

Escudero, I., \& Sánchez, V. (2007). How do domains of knowledge integrate into mathematics teachers' practice? Journal of Mathematical Behavior, 26(4), 312-327. https://doi.org/10.1016/j.jmathb.2007.11.002

Fernández, C., Callejo, M. L., \& Márques, M. (2014). Conocimiento de los estudiantes para maestro cuando interpretan respuestas de estudiantes de primaria a problemas de división-medida. Enseñanza de las Ciencias, 32(3), 407-424. https://doi.org/10.5565/rev/ensciencias. 1235

Fernández, C., \& Choy, B. H. (2020). Theoretical lenses to develop mathematics teacher noticing. Learning, teaching, psychological and social perspectives. En S. Llinares, \& O. Chapman (Eds.), International Handbook of Mathematics Teacher Education. Volume 2: Tools and Processes in mathematics Teacher Education (2nd Edition) (pp. 337-360). Brill/Sense. https://doi.org/10.1163/9789004418967013

Fernández, C., Llinares, S., \& Rojas, Y. (2020). Prospective mathematics teachers' development of noticing in an online teacher education program. ZDM Mathematics Education, 52, 959-972. https://doi.org/10.1007/s11858-020-01149-7

Fernández, C., Llinares, S., \& Rojas, Y. (2021). The impact of an online teacher education program on the development of prospective secondary mathematics teachers' noticing. En K. Hollebrands, R. Anderson, \& K. Oliver (Eds.), Online Learning in Mathematics Education (pp. 187-206). Springer Nature. https://doi.org/10.1007/978-3-030-80230-1 10 
Fernández, C., Llinares, S., \& Valls, J. (2011). Características del desarrollo de una mirada profesional en estudiantes para profesor de matemáticas en un contexto b-learning. Acta Scientiae. Revista de Ensino de Ciencias e Matemática, 13(1), 9-30.

Fernández, C., Sánchez-Matamoros, G., Valls, J., \& Callejo, M. L. (2018). Noticing students' mathematical thinking: characterization, development and contexts. Avances de Investigación en Educación Matemática (AIEM), 13, 39-61. https://doi.org/10.35763/aiem.v0i13.229

Groenwald, C. L. O., \& Llinares, S. (2019). Competencia docente de observar con sentido situaciones de enseñanza. Revista Paradigma, XL(1), 29-46. http://revistaparadigma.online/ojs/index.php/paradigma/article/view/740

Hummes, V. B., Font, V., \& Breda, A. (2019). Combined use of the lesson study and didactic suitability for the development of reflection on the own practice in the training of mathematics teachers. Acta Scientiae. Revista de Ensino de Ciencias e Matemática, 21(1), 64-82. https://doi.org/10.17648/acta.scientiae.v21iss1id4968

Ivars, P., Buforn, A., \& Llinares, S. (2016). Características del aprendizaje de estudiantes para maestro de una trayectoria de aprendizaje sobre las fracciones para apoyar el desarrollo de la competencia docente "Mirar Profesionalmente". Acta Scientiae. Revista de Ensino de Ciencias e Matemática, $18(4), 48-66$.

Ivars, P., Buforn, A., \& Llinares, S. (2017). Diseño de tareas y desarrollo de una mirada profesional sobre las situaciones de enseñanza de las matemáticas de estudiantes para maestro. En A. Salcedo (Comp.), Alternativas Pedagógicas para la Educación Matemática del siglo XXI (pp. 65-87). Centro de Investigaciones Educativas, Escuela de Educación. Universidad Central de Venezuela.

Ivars, P., Fernández, C., \& Llinares, S. (2020a). Uso de una trayectoria hipotética de aprendizaje para proponer actividades de instrucción. Enseñanza de las Ciencias, 38(3), 105-124. https://doi.org/10.5565/rev/ensciencias.2947

Ivars, P., Fernández, C., \& Llinares, S. (2020b). A learning trajectory as a scaffold for pre-service teachers' noticing of students' mathematical understanding. International Journal of Science and Mathematics Education, 18, 529-548. https://doi.org/10.1007/s10763-019-09973-4

Ivars, P., Fernández, C., Llinares, S., \& Choy, B. H. (2018). Enhancing noticing: using a hypothetical learning trajectory to improve preservice primary teachers' professional discourse. EURASIA Journal of Mathematics, Science and Technology Education, 14(11), em1599. https://doi.org/10.29333/ejmste/93421 
Jacobs, V. R., \& Spangler, D. (2017). Research on core practices in K-12 mathematics teaching. En J. Cai (Ed.), COMPEDIUM for Research in Mathematics Education (pp.766-792). NCTM.

Jaime, A., \& Gutiérrez, A. (1990). Una propuesta de fundamentación para la enseñanza de la geometría: El modelo de Van Hiele. En S. Llinares, \& M. V. Sánchez (Eds.), Teoría y práctica en educación matemática (pp. 295-384). Editorial Alfar.

Le Boterf, G. (1994). De la compétence: Essai sur un atracteur étrange. Les Éditión D’ Organization.

Llinares, S. (2013a). El desarrollo de la competencia docente “mirar profesionalmente” la enseñanzaaprendizaje de las matemáticas. Educar em Revista, 50, 117-133.

https://doi.org/10.1590/S0104-40602013000400009

Llinares, S. (2013b). Professional noticing: a component of the mathematics teacher's professional practice. SISYPHUS, 1(3), 76-93.

Llinares, S., \& Fernández, C. (2021). Mirar profesionalmente la enseñanza de las matemáticas: características de una agenda de investigación en Didáctica de la Matemática. La GACETA de la RSME, 24(1), 185-205.

Llinares, S., Ivars, P., Buforn, A., \& Groenwald, C. L. O. (2020). "Mirar Profesionalmente” las situaciones de enseñanza: una competencia basada en el conocimiento. En E. Badillo, N. Climent, C. Fernández, \& M. T. González (Eds.), Investigación sobre el profesor de matemáticas: formación, práctica de aula, conocimiento y competencia profesional (pp. 177-192). Ediciones Universidad Salamanca.

Mason, J. (2002). Researching your own practice: the discipline of noticing. Routledge-Falmer. https://doi.org/10.4324/9780203471876

Mason, J. (2021). Learning about noticing, by, and through, noticing. ZDM Mathematics Education, 53, 231-243. https://doi.org/10.1007/s11858-020-01192-4

Moreno, M., \& Llinares, S. (2018). Prospective mathematics teachers' perspectives on technology. En M. E. Strutchens, R. Huang, D. Potari, \& L. Losano (Eds.), Educating Prospective Secondary Mathematics Teachers, ICME-13 Monographs (pp. 125-142). Springer Nature. https://doi.org/10.1007/978-3-319-91059-8 8

Moreno, M., Sánchez-Matamoros, G., Callejo, M. L., Perez-Tyteca, P., \& Llinares, S. (2021). How prospective kindergarten teachers develop their noticing skills: the instrumentalization of a learning trajectory. ZDM Mathematics Education, 53, 57-72. https://doi.org/10.1007/s11858-021-01234-5 
NCTM. (2015). De los principios a la acción-para garantizar el éxito matemático para todos. Editando libros S.A.

Penalva, M. C., \& Llinares, S. (2011). Tareas matemáticas en la Educación Secundaria. En J. M. Goñi (Ed.), Didáctica de las Matemáticas (pp. 27-52). Grao.

Perrenoud, P. (2000). 10 novas competências para ensinar. ARTMED.

Sánchez-Matamoros García, G., Moreno Moreno, M., Pérez Tyteca, P., \& Callejo de la Vega, M. L. (2018). Trayectoria de aprendizaje de la longitud y su medida como instrumento conceptual usado por futuros maestros de educación infantil. Revista Latinoamericana de Investigación en Matemática Educativa, 21(2), 203-228. https://doi.org/10.12802/relime.18.2124

Sánchez-Matamoros, G., Moreno, M., \& Valls, J. (2021). Instrumental genesis of a learning trajectory: the case of Pedro's professional noticing. Acta Scientiae. Revista de Ensino de Ciencias e Matemática, 23(7), 91-119, https://doi.org/10.17648/acta.scientiae.6296

Schack, E. O., Fisher, M. H., \& Wilhelm, J. A. (Eds.) (2017). Teacher noticing: bridging and broadnening perspectives, contexts, and frameworks. Springer Nature. https://doi.org/10.1007/978-3-319-46753-5

Scheiner, T. (2021). Towards a more comprehensive model of teacher noticing. ZDM Mathematics Education, 53, 85-94, https://doi.org/10.1007/s11858-020-01202-5

Seibert, L. G., Groenwald, C. L. O., \& Llinares, S. (2013). Observar com sentido: uma competência importante na vida professional do professor de matemática. Acta Scientiae. Revista de Ensino de Ciencias e Matemática, 15(1), 133-152.

Silva, M. R. da. (2008). Currículo e competências: a formação administrada. Cortez.

Stahnke, R., Schueler, S., \& Roesken-Winter, B. (2016). Teachers' perception, interpretation, and decision-making: a systematic review of empirical mathematics education research. ZDM Mathematics Education, 48(1-2), 1-27, https://doi.org/10.1007/s11858-016-0775-y

Stein, M. K., Grover, B. W., \& Henningen, M. (1996). Building student capacity for mathematical thinking and reasoning: an analysis of mathematical tasks used in reform classrooms. American Educational Research Journal, 33, 455-488. https://doi.org/10.3102/00028312033002455

van Es, E. A., \& Sherin, M. G. (2002). Learning to notice: scaffolding new teachers' interpretations of classroom interactions. Journal of Technology and Teacher Education, 10(4), 571-596. 
van Es, E. A., \& Sherin, M. G. (2021). Expanding on prior conceptualizations of teacher noticing. ZDM Mathematics Education, 53, 17-27. https://doi.org/10.1007/s11858-020-01211-4

\section{Cómo citar este artículo:}

Groenwald, C. L. O., \& Llinares, S. (2022). Aprendiendo a mirar profesionalmente las situaciones de enseñanza de las matemáticas. Revista Venezolana de Investigación en Educación Matemática (REVIEM), 2(2), e202202. https://doi.org/10.54541/reviem.v2i2.29 


\section{Claudia Lisete O. Groenwald, Salvador Llinares}

cC) (†)

Copyright ( 2022 . Claudia Lisete O. Groenwald, Salvador Llinares. Esta obra está protegida por una licencia Creative Commons 4.0. International (CC BY 4.0).

Usted es libre para Compartir — copiar y redistribuir el material en cualquier medio o formato - y Adaptar el documento - remezclar, transformar y crear a partir del material — para cualquier propósito, incluso para fines comerciales, siempre que cumpla la condición de:

Atribución: Usted debe dar crédito a la obra original de manera adecuada, proporcionar un enlace a la licencia, e indicar si se han realizado cambios. Puede hacerlo en cualquier forma razonable, pero no de forma tal que sugiera que tiene el apoyo del licenciante o lo recibe por el uso que hace de la obra.

$\underline{\text { Resumen de licencia - Texto completo de la licencia }}$ 